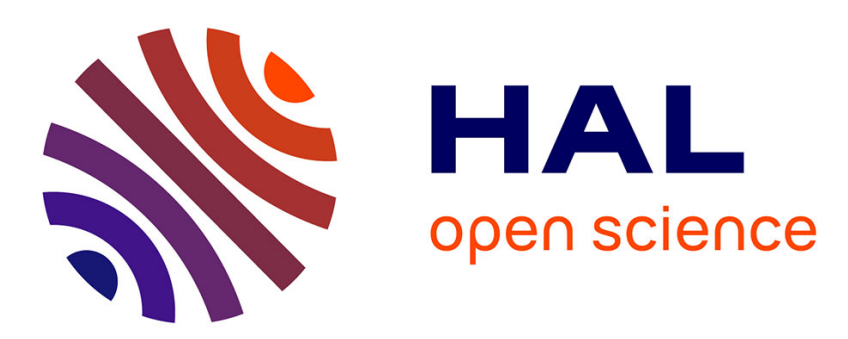

\title{
Improved solutions for ill-conditioned problems involved in set-membership estimation for fault detection and isolation
}

\author{
Laleh Hosseini-Ravanbod, Carine Jauberthie, Nathalie Verdière, Louise \\ Travé-Massuyès
}

\section{To cite this version:}

Laleh Hosseini-Ravanbod, Carine Jauberthie, Nathalie Verdière, Louise Travé-Massuyès. Improved solutions for ill-conditioned problems involved in set-membership estimation for fault detection and isolation. Journal of Process Control, 2017, 58, pp.139 - 151. 10.1016/j.jprocont.2017.08.003 . hal01740541

\author{
HAL Id: hal-01740541 \\ https://hal.science/hal-01740541
}

Submitted on 21 Nov 2018

HAL is a multi-disciplinary open access archive for the deposit and dissemination of scientific research documents, whether they are published or not. The documents may come from teaching and research institutions in France or abroad, or from public or private research centers.
L'archive ouverte pluridisciplinaire HAL, est destinée au dépôt et à la diffusion de documents scientifiques de niveau recherche, publiés ou non, émanant des établissements d'enseignement et de recherche français ou étrangers, des laboratoires publics ou privés. 


\title{
Improved solutions for ill-conditioned problems involved in set-membership estimation for fault detection and isolation
}

\author{
Laleh Ravanbod \\ Université du Havre; 25 rue Philippe Lebon; BP 540; 76058 Le Havre Cedex, France \\ Carine Jauberthie \\ CNRS, LAAS, 7, avenue du Colonel Roche, F-31400 Toulouse, France \\ Université de Toulouse; UPS, LAAS; F-31400 Toulouse, France \\ Nathalie Verdière \\ Normandie Univ, UNIHAVRE, LMAH, FR-CNRS-3335, ISCN, 76600 Le Havre, France \\ Louise Travé-Massuyès \\ CNRS, LAAS, 7, avenue du Colonel Roche, F-31400 Toulouse, France \\ Université de Toulouse; LAAS; F-31400 Toulouse, France
}

\begin{abstract}
Set-membership (SM) estimation implies that the computed solution sets are guaranteed to contain all the feasible estimates consistent with the bounds specified in the model. Two issues often involved in the solution of SM estimation problems and their application to engineering case studies are considered in this paper. The first one is the estimation of derivatives from noisy signals, which in a bounded uncertainty framework means obtaining an enclosure by lower and upper bounds. In this paper, we improve existing methods for enclosing derivatives using Higher-Order Sliding Modes (HOSM) differentiators combining filtering. Our approach turns the use of high order derivatives more efficiently especially when the signal to differentiate has slow dynamics. The second issue of interest is solving linear interval equation systems, which is often an ill-conditioned problem. This problem is reformulated as a Constraint Satisfaction Problem and solved by the combination of the constraint propagation Forward
\end{abstract}

\footnotetext{
*Corresponding author

Email address: c jaubertelaas.fr (Carine Jauberthie)
}

Preprint submitted to Journal of Process Control

August 23, 2017 
Backward algorithm and the SIVIA algorithm. The two proposed methods are tested on illustrative examples. The two methods are then used in a fault detection and isolation algorithm based on SM parameter estimation that is applied to detect abnormal parameter values in a biological case study.

Keywords: , Uncertain dynamic systems, Bounded noise, Nonlinear systems, Conditioning problem, Fault Detection and Diagnosis

\section{Introduction}

This paper focuses on two mathematical problems encountered in several engineering problems: the calculus of uncertain derivatives and conditioning problems involved in solving linear interval equation systems. These two issues are addressed in 5 a set-membership (SM) framework in which uncertainties are characterized by simple bounds. SM estimation methods advantageously provide guaranteed solutions, meaning that the computed set estimates are guaranteed to contain all the feasible estimates consistent with the specified bounds. SM estimation methods have been successfully applied to many tasks ([1, 2, 3, 4]). SM estimation can be based on interval analysis that was introduced by [5] and several algorithms have been proposed along this line for nonlinear systems (for more details, see [6, 4, 7]). The approaches dedicated to linear systems are rather based on ellipsoid shaped methods (for example [8]), parallelotope or zonotope based methods [9]. The advantage of providing guaranteed results is unfortunately often stained by the overestimation of the results. In this respect, it is mandatory to carefully analyse every single step of SM estimation methods to compensate for possible spurious uncertainty propagation. In particular, the resolution of estimation problems often requires to evaluate successive derivatives of signals, which is known to be a tedious numerical problem, and/or to solve systems of linear interval equations, which must often handle ill-conditionment. This paper deeply inspects these two problems and proposes improved solution methods.

Derivative estimation from noisy signals given by discrete measurement samples is an important and difficult task in numerical analysis, signal processing and control. It is well-known that it is an ill-posed problem. In the literature, several classes of derivative estimation methods have been proposed. The first class consists in approximating 

criterion [10, 11]. Another class consists in approximating the signal by a truncated Taylor expansion and to operate in either the Mikusinski field [12, 13] or the distribution space [14]. Yet another class is based on sliding-mode differentiators [15, 16]. In the frame of interval analysis, apart from the classical finite difference that has been extended [17], there are few works guaranteeing enclosures of successive derivatives. Nevertheless, the estimation of derivatives is essential in many basic algorithms such as the evaluation of centered inclusion functions, Newton contractors, etc. In [18], the Higher-Order Sliding Modes (HOSM) differentiators developed by Levant in 1998 have been used to obtain an exact enclosure of derivatives required by a fault detection method. The drawback of these differentiators is that only the first derivative is calculated with reasonable overestimation contrary to higher order derivatives. In this paper, we propose to combine the methods developed by Levant with a zero-phase low-pass filtering algorithm, guaranteeing a robust enclosure of the successive derivatives even for high orders. Some examples are given and confirm the robustness of the method.

40

The problem of solving a linear interval system is considered in the second part of the paper. Although the system is linear, this problem is NP-hard due to the presence of interval matrices [19]. Some algorithms for solving interval linear systems return a box containing the convex hull of the solutions, which is not the minimal enclosure [21, 22], we rely on an iterative method [20] because it advantageously allows one to control the computation time. Our method assumes that an initial enclosure is known from the knowledge of the system and applies contractions using the Forward Backward Propagation algorithm [23] based on interval Gauss-Seidel iterations. Then we use this solution to initialize a branch and bound algorithm, based on Set Inversion Via Interval Analysis (SIVIA), which further improves the result. Some details on contractors and the SIVIA algorithm can be found in [23], [6] and [24].

The last part of the paper integrates the two proposed methods to improve an off55 
SM parameter estimation [25]. The developed FDI procedure requires the estimation of derivatives, sometimes of high order, from discrete measurement samples and the solution of linear systems of interval equations involving blocks of parameters.

The paper is organized as follows. Section 2 briefly introduces the problem of enclosing successive derivatives of a signal corrupted by bounded noise and the new method that we propose based on HOSM differentiation and filtering is presented. Bounded noises are a natural way to model the realistic stochastic fluctuations of a biological system, for example, that are caused by its interaction with the external world. Bounded noise is also well-adapted to sensors tolerances. The proposed method is applied to classical examples which highlight its advantages. Section 3 explains the problem of solving linear interval systems, and exhibits the sources of ill-conditioning. Our method based on contractors and set inversion is presented. Through some examples, the results obtained by the proposed scheme are compared with those obtained by classical solvers. In section 4, the previously developed methods are applied in a SM algorithm for FDI in nonlinear dynamical systems. The application of this algorithm to a cell exchange model is reported. Finally, section 5 concludes the paper and provides some perspectives.

\section{Derivative estimation}

The aim of this section is to present a differentiator that provides robust exact intervals containing the successive derivatives of a signal corrupted by a bounded noise whose bounds are supposed known. Bounded noise is a convenient way to characterize uncertainty when a more informative statistical model is not known. It accounts for nonlinear phenomena like saturation which are often encountered in practice. For example, bounded noise is a natural way to model the realistic stochastic fluctuations of a biological system, for example, that are caused by its interaction with the external world [26].

The differentiator has been proposed by Levant in 1998 [15] under the name of the Higher-Order Sliding Modes (HOSM) differentiator. It is presented in the first part 
of this section. Although other differentiators like the asymptotic differentiator [27] or the high gain observer [28] have been proposed, the differentiator of [15] advantageously provides exact differentiation in finite-time of noise-free signals satisfying some Lipschitz constraint. That is why we have built on this work.

Despite its advantages, the Higher-Order Sliding Modes (HOSM) differentiator has the drawback that for a high derivative order or for a significant noise, the intervals containing the derivative estimates are very overestimated. Thus we propose an original approach that provides tighter interval enclosures of derivatives thanks to a low pass filter. This smoothing makes the enclosure of high order derivatives more efficient specially when the signal to be differentiated has slow dynamics.

Let us consider the following standard notations and definitions [6]. A real interval $[x]=[\underline{x}, \bar{x}]$ is a connected and closed subset of $\mathbb{R}$. The notation $\mathbf{x}$ defines the real vector $\mathbf{x}=\left(x_{1}, \ldots, x_{n}\right)^{T}$, where ${ }^{T}$ stands for the transpose of the considered vector whereas $[\mathbf{x}]$ defines an interval vector, also called a box. $w$ represents the interval width. If $[x]=[\underline{x}, \bar{x}]$ then $w([x])=\bar{x}-\underline{x}$. In the same manner $w([\mathbf{x}])=\max (\overline{\mathbf{x}}-\underline{\mathbf{x}})$.

\subsection{HOSM differentiator}

In the works [15], [29], [16] concerning HOSM differentiators, the signal $y(t)$ to be differentiated is considered as a function defined on $[0,+\infty[$. It is supposed to be composed of a bounded Lebesgue-measurable noise $e(t)$ (bounded by a positive constant $\alpha$ ) with unknown features and an unknown base signal $y_{0}(t)$ with the $m^{t h}$ derivative having a known Lipschitz constant $C>0$. The $m$ successive derivatives of the signal $y(t)$, i.e. $y^{(1)}(t), \ldots, y^{(m)}(t)$ are estimated by $z_{1}(t), \ldots, z_{m}(t)$ for $t \geq t_{c}$ as 
described below:

$$
\left\{\begin{aligned}
\dot{z}_{0}= & v_{0}, \\
v_{0}= & -\lambda_{0}\left|z_{0}-y\right|^{\frac{m}{(m+1)}} \operatorname{sign}\left(z_{0}-y\right)+z_{1}, \\
\dot{z}_{1}= & v_{1}, \\
v_{1}= & -\lambda_{1}\left|z_{1}-v_{0}\right|^{\frac{(m-1)}{m}} \operatorname{sign}\left(z_{1}-v_{0}\right)+z_{2}, \\
\vdots & \\
\dot{z}_{m-1}= & v_{m-1}, \\
v_{m-1}= & -\lambda_{m-1}\left|z_{m-1}-v_{m-2}\right|^{\frac{1}{2}} \operatorname{sign}\left(z_{m-1}-v_{m-2}\right) \\
& +z_{m}, \\
\dot{z}_{m}= & -\lambda_{m} \operatorname{sign}\left(z_{m}-v_{m-1}\right),
\end{aligned}\right.
$$

where $\lambda_{j} \in \mathbb{R}, j=0, \ldots, m$, represent the differentiator parameters and $t_{c}$ is the convergence time of the differentiator which depends on $\lambda_{j}$. Generally, the parameters $\lambda_{j}, j=0, \ldots, m$, are chosen experimentally (for more details, see [15], [29]). In other words, these parameters must be re-evaluated for every new signal. This is not surprising because it is well known that an ideal differentiator, i.e. that can differentiate any signal, does not exist.

In the case of an additive bounded measurement noise, i.e. $y(t)=y_{0}(t)+e(t)$ with $|e(t)| \leq \alpha$, Levant [29] proposes the following theorem:

Theorem 1 ([29]). The following inequalities hold after a finite convergence time $t_{c}$ for some positive constants $\eta_{k}$ depending exclusively on the parameters of the differentiator:

$$
\left|z_{k}(t)-y_{0}^{(k)}(t)\right| \leq \eta_{k} \alpha^{\frac{m+1-k}{m+1}}, \quad k=1,2, \ldots, m .
$$

Therefore, if $z_{k}$ is computed by 11 , then the interval containing the $k^{t h}$ exact derivative of $y_{0}(t)$, i.e. $\left[y_{0}^{(k)}(t)\right]$, is given by:

$$
\left[y_{0}^{(k)}(t)\right]=\left[z_{k}(t)-a c c_{k}, \quad z_{k}(t)+a c c_{k}\right], \quad k=1,2, \ldots, m \quad t \geq t_{c},
$$

where $a c c_{k}=\eta_{k} \alpha^{\frac{m+1-k}{m+1}}$, and $\eta_{k}$ and the convergence time $t_{c}$ depend exclusively on $\lambda_{j}, j=0, \ldots, m$. 
Thus if $z_{k}$ is computed by 11 , then the interval containing the $k^{t h}$ exact derivative of $y(t)$ is given by:

$$
\left[z_{k}(t)-a c c_{k}, z_{k}(t)+a c c_{k}\right]
$$

Another way to tighten $\left[y_{0}^{(k)}(t)\right]$ is to cascade the differentiator with a low-pass filter and that is the approach that is proposed here. It is first shown that the bounds obtained with the proposed method are also rigorous and guaranteed.

The inequality $(3)$ in discrete time form is given by:

$$
z_{k}(n)-a c c_{k} \leq y_{0}^{(k)}(n) \leq z_{k}(n)+a c c_{k}, \quad n \geq n_{c},
$$

where $z_{k}(n):=z_{k}\left(n T_{s}\right), y_{0}^{(k)}(n):=y_{0}^{(k)}\left(n T_{s}\right), T_{s}$ is the sampling period and the convergence sample time $n_{c}$ is given by the ceil of $t / T_{s}$.

For any positive integer number $K$, the following relation also holds:

$$
z_{k}(n)-a c c_{k} \leq y_{0}^{(k)}(n) \leq z_{k}(n)+a c c_{k}, \quad n_{c} \leq n \leq K+n_{c}
$$

which can be rewritten as the following $K$ relations:

$$
z_{k}\left(n_{c}+i\right)-a c c_{k} \leq y_{0}^{(k)}\left(n_{c}+i\right) \leq z_{k}\left(n_{c}+i\right)+a c c_{k}, \quad i=1, \ldots, K .
$$

Averaging through these $K$ relations and remembering that $a c c_{k}$ is constant, we obtain

$$
\frac{1}{K} \sum_{i=1}^{K} z_{k}\left(n_{c}+i\right)-a c c_{k} \leq \frac{1}{K} \sum_{i=1}^{K} y_{0}^{(k)}\left(n_{c}+i\right) \leq \frac{1}{K} \sum_{i=1}^{K} z_{k}\left(n_{1}+i\right)+a c c_{k} .
$$


Assume that $y_{0}^{(k)}(n)$ is sufficiently slow-varying, then the HOSM output $z_{k}(n)$ is also slow-varying. In this case, the terms $\frac{1}{K} \sum_{i=1}^{K} y_{0}^{(k)}\left(n_{c}+i\right)$ and $\frac{1}{K} \sum_{i=1}^{K} z_{k}\left(n_{c}+i\right)$ are in fact filtered versions of $y_{0}^{(k)}\left(n_{c}+i+1\right)$ and of $z_{k}\left(n_{c}+i+1\right)$, respectively. The filter is a low-pass FIR filter of order $K$. Finally, using the notation $\mathcal{F}($.$) for the filtered$ signal, relation 6 can be rewritten as

$$
\mathcal{F}\left(z_{k}(n)\right)-a c c_{k} \leq \mathcal{F}\left(y_{0}^{(k)}\right)(n) \leq \mathcal{F}\left(z_{k}(n)\right)+a c c_{k}, \quad n \geq n_{c}+K+1,
$$

showing that the bounds are really guaranteed outer bounds.

Unfortunately, the proposed method rises some issues. It is well known that every realizable (causal) filter introduces phase and hence time delay. Among all causal lowpass filters, it is only for low-pass Finite-Impulse-Response (FIR) filters that the delay can be computed explicitly because the phase of this filter is linear with respect to the frequency. In fact, there exists a direct relationship between the number of parameters of the filter and the delay. However, the drawback of FIR filters is their large number of parameters. Indeed, evaluating each filtered sample requires $K$ previous samples or in other words $K . T_{s}$ seconds delay. In addition, for higher order derivatives, the input noise is further amplified and then $K$ must be extremely high to filter it efficiently, which means reducing the cut-off frequency of the low-pass filter. However, very low pass-band removes essential information of the signal. The trade-off between the maximum derivation order and the minimum cut-off frequency is much easier to achieve when the signal to be differentiated is already slow-varying. In this case, it is sure that even high order derivatives remain low-pass. This trade-off can be achieved experimentally and generally leads to higher $K s$ for the successive derivatives. This means that higher order derivatives may involve higher time delays. This can be harmful for the use in an FDI method (cf. Section 4p: first because the inherent time delay of the method may not be acceptable for online FDI applications, second because the Analytical Redundant Relations to be evaluated for FDI involve several higher order derivatives (cf. Section 4), which rises a difficult synchronization problem.

${ }_{145}$ To prevent the delay and synchronization problems caused by a causal filter, a non causal low-pass filter can be used. However, because such filter cannot be implemented online, one must then accept to run the FDI application offline. We propose to use a 
class of low-pass filters called zeros-phase low-pass digital filters. These low-pass filters are not causal but they are numerically implementable and they allow one to eliminate the synchronization issue by processing discrete input data in both the forward and inverse directions. They also minimize start-up and ending transients by matching initial conditions. The FDI method presented in Section (4) uses this kind of filter and is applied offline.

\subsection{Examples of derivative estimation enclosure}

Hereafter, a second-order zero-phase Butterworth digital filter with cutoff frequency $5 \mathrm{~Hz}$ is used. Butterworth filters are characterized by a magnitude response that is maximally flat in the pass-band and monotonic overall [30]. The sampling period is chosen as $T_{s}=10^{-4} \mathrm{~s}$. The simulations are carried out with the 2nd-order Runge-Kutta method with an integration step equal to $10^{-4} \mathrm{~s}$.

In the following examples taken from [15], we compare the results obtained with the differentiator of Levant [15] and with the improved version of this differentiator that we propose.

Example 2.1. Let $y(t)=\sin (t)+5 t+1$ be a noiseless signal to be differentiated. The parameters of the differentiator are chosen as $\lambda_{0}=2, \lambda_{1}=6$ and $\lambda_{2}=8$.

Figure 1 displays the first and second derivatives.
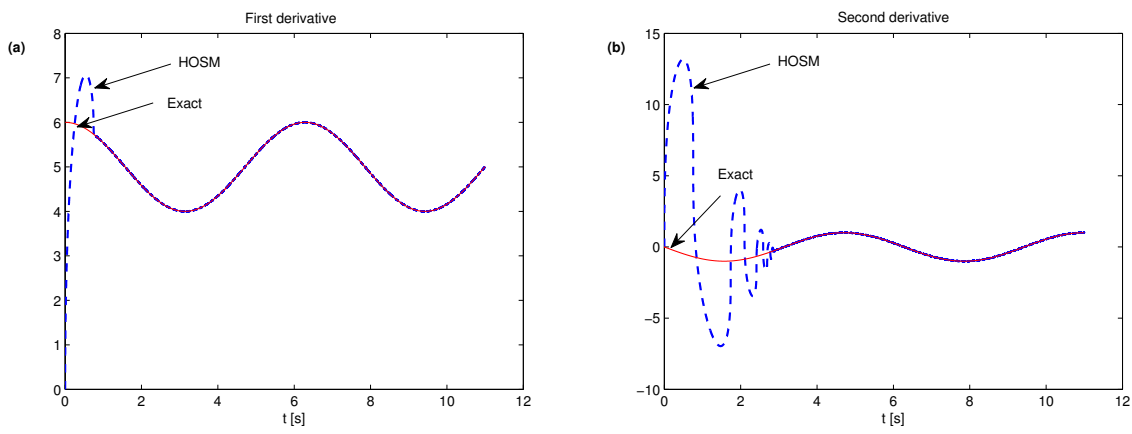

Figure 1: First derivative (left) and second derivative (right) for Example 2.1 
Example 2.2. Consider now the noisy signal $y(t)=y_{0}(t)+e(t)=\sin (t)+5 t+1+$ $e(t)$ with $|e(t)| \leq \alpha=0.04$ to be differentiated. The variables $\eta_{1}$ and $\eta_{2}$ are given by $\eta_{1}=2.1211$ and $\eta_{2}=8.5136$. The other parameters are the same as in the last example.

Figure 2 represents the derivative enclosures obtained with HOSM and Figure 3 represents the derivative enclosures obtained with our method that includes a filter. In these figures, the dashed black line stands for the exact noise free derivative while light and dark gray lines represent the upper and lower bounds respectively. The width reduces from $2 \times 0.52$ to $2 \times 0.23$ for the first derivative and from $2 \times 5$ to $2 \times 2.9$ for the second derivative. In other words, the widths are reduced by $55.8 \%$ and $42 \%$ for the first and the second derivative, respectively.
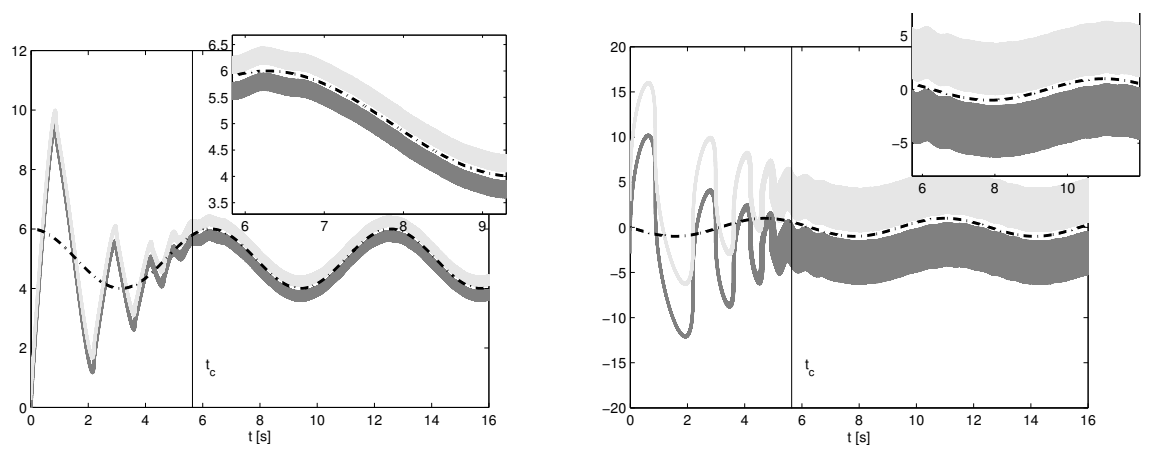

Figure 2: First derivative enclosure (left) and second derivative enclosure (right) with HOSM for Example 2.2
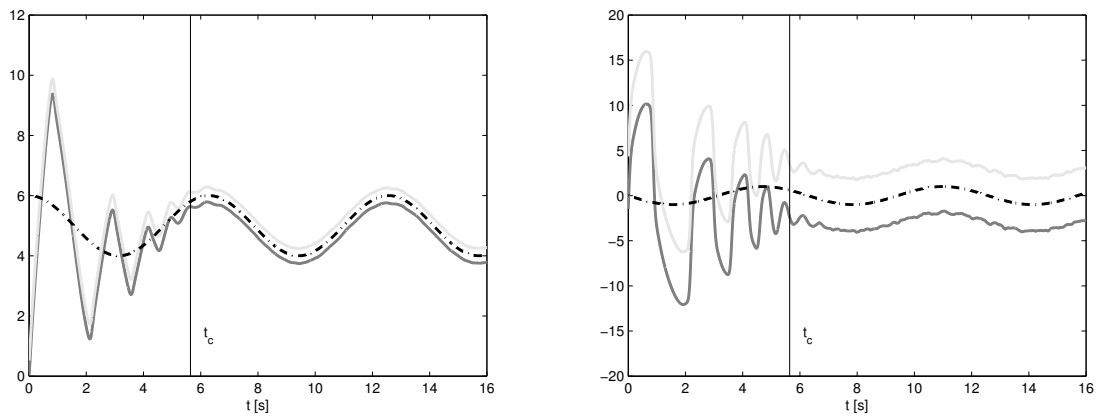

Figure 3: First derivative enclosure (left) and second derivative enclosure (right) with our method for Example 2.2 
These figures show the efficiency of the proposed approach to tighten the enclosure intervals without changing the convergence time $t_{c} \approx 5.8 s$, particularly for the second derivative.

Example 2.3. This example considers determining the five first derivative enclosures for the signal $y(t)=y_{0}(t)+e(t)$, where $y_{0}(t)=\sin (t)+5 t$ and $|e(t)| \leq \alpha=10^{-6}$. Figure 4 represents the five enclosures of the five first derivatives of $y_{0}(t)$ obtained by using the HOSM differentiator cascaded with a filter. As it can be seen, the filter does not introduce any delay and $t_{c}$ remains about 2.28 seconds.
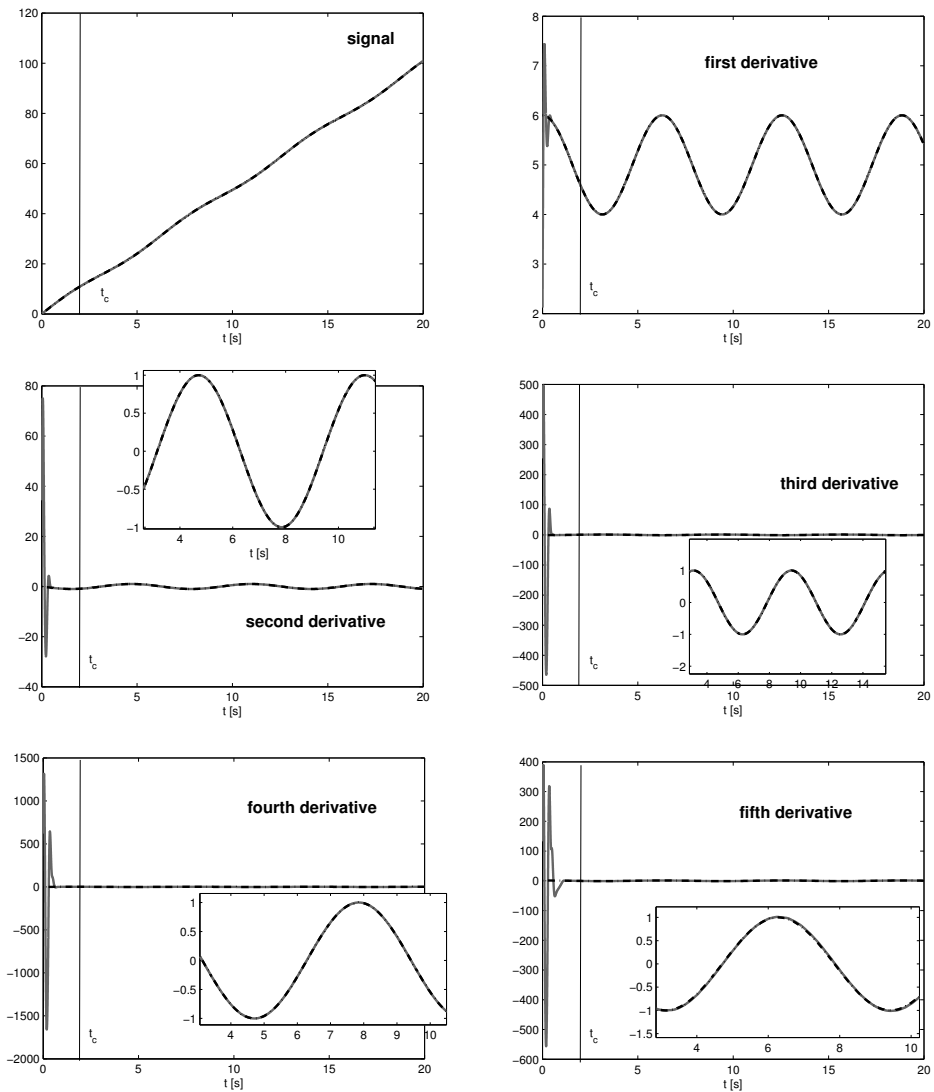

Figure 4: Derivative enclosures with our method for Example 2.3 noiseless case.

Figure 5 represents the five enclosures of the five derivatives of $y(t)$ obtained by using the HOSM differentiator. The fourth and fifth derivatives are unusable. 

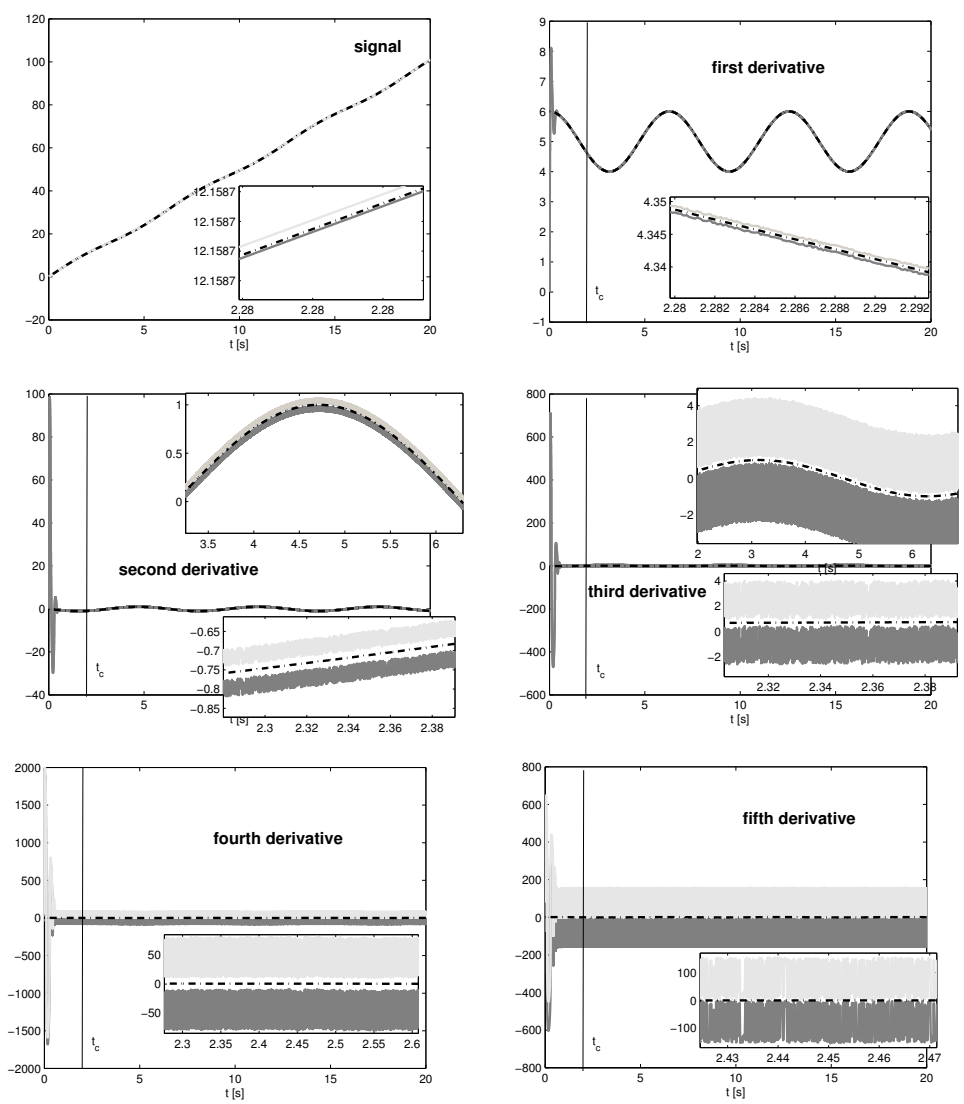

Figure 5: Derivative enclosures with HOSM for Example 2.3 noisy case.

Figure 6 represents the envelopes obtained by our approach. By using this ap190 proach, we obtain a meaningful envelopes even for the fifth derivative and $t_{c}=2.28$ is always preserved everywhere. 

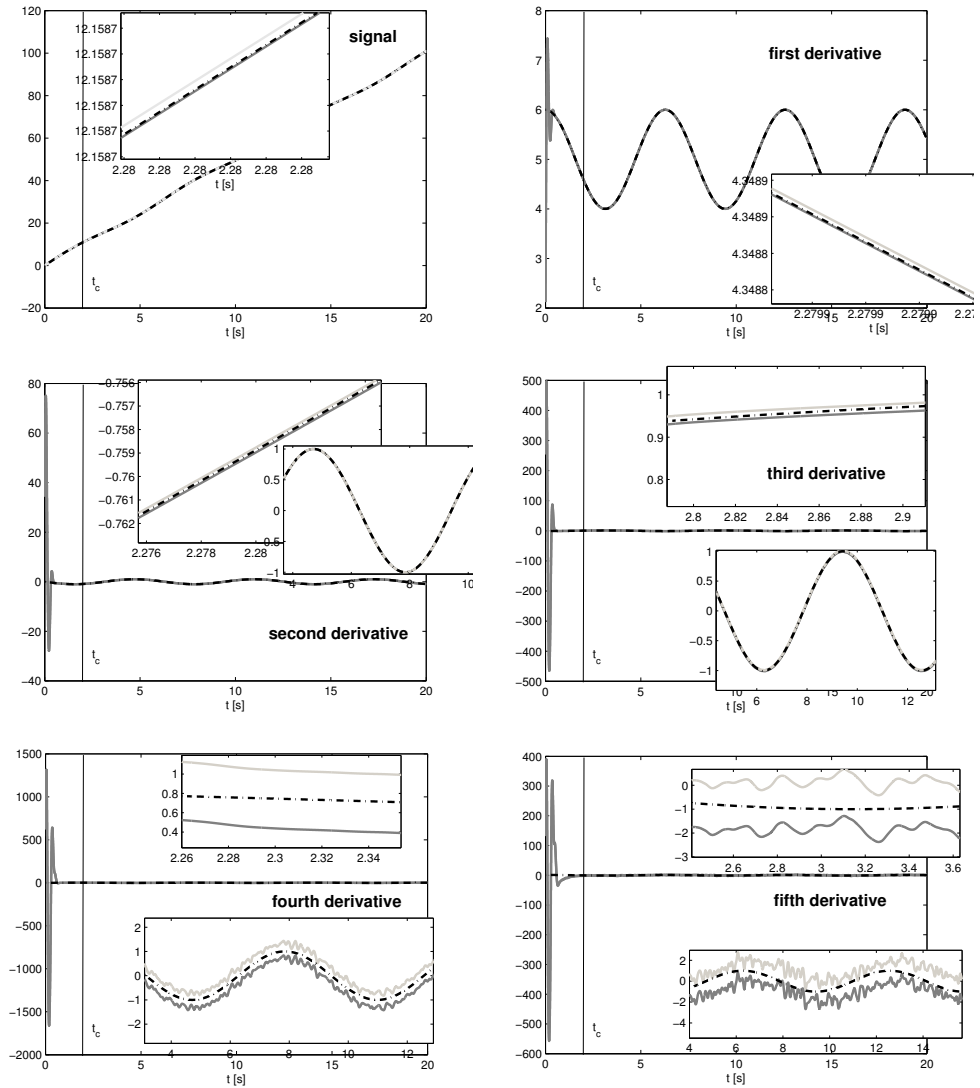

Figure 6: Derivative enclosures with our method for Example 2.3. noisy case.

The next section switches to another important and common issue, which echoes many practical problems. It consists in solving systems of interval linear equations. Like the previously considered problem of robust exact differentiation, this problem is also involved in the FDI method based on SM parameter estimation presented in Section 4

\section{Linear systems of interval equations}

Interval analysis was first introduced [5] to take into account the quantification errors introduced by the floating point representation of real numbers in computers. Later, it has been extended to validate numeric procedures. Indeed, interval analysis 
permits to obtain guaranteed results in the sense that they enclose all the results consistent with the accounted uncertainty, and this in a finite number of operations. Very naturally, methods based on interval analysis have hence been applied for the resolution of linear systems [31].

A linear system of interval equations $[\mathbf{A}][\mathbf{x}]=[\mathbf{b}]$, where $[\mathbf{A}]$ is an $(m \times n)$ dimensional interval matrix and is not hence necessarily a square matrix, can be rewritten as $0 \in[\mathbf{A}][\mathbf{x}]-[\mathbf{b}]$. Classically, solving such systems is formulated as a set inversion problem [6]. In its general form, the set inversion problem consists in determining the solution set $S$ for the unknown quantities $\mathrm{x}$ defined by:

$$
S=\left\{\mathbf{x} \in \mathbb{R}^{n} \mid f(\mathbf{x}) \in[\mathbf{y}]\right\}=f^{-1}([\mathbf{y}]),
$$

where $f$ is a possibly nonlinear function from $\mathbb{R}^{n}$ to $\mathbb{R}^{m}$ and $\mathbf{y}$ is known a priori and is a subset of $\mathbb{R}^{m}$. If $f$ is linear, Equation (8) involves computing an inverse or pseudoinverse matrix. This is a set inversion problem which can be solved using the recursive algorithm SIVIA [24]. This algorithm explores all the search space without loosing any solution and makes it possible to derive a guaranteed enclosure of the solution set $S$ such that

$$
\underline{S} \subseteq S \subseteq \bar{S}
$$

The inner enclosure $\underline{S}$ is composed of the boxes that have been proved feasible. To prove that a box $[\mathbf{x}]$ is feasible it is sufficient to prove that $f([\mathbf{x}]) \subseteq[\mathbf{y}]$. Reversely, if it can be proved that $f([\mathbf{x}]) \cap[\mathbf{y}]=\emptyset$, then the box $[\mathbf{x}]$ is unfeasible. Otherwise, no conclusion can be reached and the box $[\mathbf{x}]$ is said undetermined. The latter is then bisected in two sub-boxes that are tested if their size is greater than a user-specified precision threshold $\varepsilon>0$. Such a termination criterion ensures that SIVIA terminates after a finite number of iterations.

In the following section, we propose a method to solve interval equation systems that involves SIVIA but proposes an important preliminary step to initialize it properly. 
In interval analysis, the problem $[\mathbf{A}][\mathbf{x}]=[\mathbf{b}]$, where $[\mathbf{A}]$ is square or not, is equivalent to the problem $0 \in[\mathbf{A}][\mathbf{x}]-[\mathbf{b}]$ denoted Problem 1 in the following. Problem 1 can be solved directly by using the SIVIA algorithm [24]. The obtained solution consists in a list of solution boxes denoted $\{[\mathbf{x}]\}^{1}$ that includes feasible and undetermined boxes, providing an outer enclosure of the solution set. The proposed algorithm is, on the contrary, based on the solution of the two following problems 1 .

- Problem 2: $0 \in[\mathbf{A}][\mathbf{x}]-]-\infty, \overline{\mathbf{b}}]$,

- Problem 3: $0 \in[\mathbf{A}][\mathbf{x}]-[\underline{\mathbf{b}},+\infty[$,

where $\underline{\mathbf{b}}$ and $\overline{\mathbf{b}}$ are the lower and upper bounds of $[\mathbf{b}]$, i.e. $[\mathbf{b}]=[\underline{\mathbf{b}}, \overline{\mathbf{b}}]$.

25 It can indeed be easily shown that any solution $\left[\mathrm{x}^{*}\right]$ of Problem 1 is a solution of Problem 2 and Problem 3 and conversely:

$$
\begin{aligned}
& {\left.\left.[\mathbf{A}]\left[\mathbf{x}^{*}\right]=[\mathbf{b}]=[\underline{\mathbf{b}}, \overline{\mathbf{b}}]=\right]-\infty, \overline{\mathbf{b}}\right] \cap[\underline{\mathbf{b}},+\infty[} \\
\Leftrightarrow & \left.0 \in[\mathbf{A}]\left[\mathbf{x}^{*}\right]-(]-\infty, \overline{\mathbf{b}}\right] \cap[\underline{\mathbf{b}},+\infty[) \\
\Leftrightarrow & \left.\left.0 \in\left([\mathbf{A}]\left[\mathbf{x}^{*}\right]-\right]-\infty, \overline{\mathbf{b}}\right]\right) \cap\left([\mathbf{A}]\left[\mathbf{x}^{*}\right]-[\underline{\mathbf{b}},+\infty[)\right. \\
\Leftrightarrow & \left.\left.0 \in\left([\mathbf{A}]\left[\mathbf{x}^{*}\right]-\right]-\infty, \overline{\mathbf{b}}\right]\right) \text { and } 0 \in\left([\mathbf{A}]\left[\mathbf{x}^{*}\right]-[\underline{\mathbf{b}},+\infty[) .\right.
\end{aligned}
$$

Another way to show the equivalence is to formulate the three problems in terms of linear inequalities:

$$
\begin{aligned}
& -\infty \leq[\mathbf{A}]\left[\mathbf{x}^{*}\right] \leq \overline{\mathbf{b}} \\
& \text { and } \\
& \underline{\mathbf{b}} \leq[\mathbf{A}]\left[\mathbf{x}^{*}\right] \leq \infty
\end{aligned} \Leftrightarrow \underline{\mathbf{b}} \leq[\mathbf{A}]\left[\mathbf{x}^{*}\right] \leq \overline{\mathbf{b}}
$$

The algorithm hence consists in the two following steps.

\footnotetext{
${ }^{1}$ In practice, these two problem are posed as:

- Problem 2: $0 \in[\mathbf{A}][\mathbf{x}]-\left[-a I_{n \times 1}, \overline{\mathbf{b}}\right]$,

- Problem 3: $0 \in[\mathbf{A}][\mathbf{x}]-\left[\underline{\mathbf{b}}, a I_{n \times 1}\right]$, where $a \gg 0$ and $I_{n \times 1}$ is the identity matrix of dimensions $n$ by 1 .
} 
- Step 1: Given a large initial box $\left[\mathbf{x}_{\text {initial }}\right]$, use the Forward Backward Propaga-

Example 3.1. In the first example, we consider the linear system:

$$
\left\{\begin{array}{l}
2 x_{1}+4 x_{2}=8, \\
x_{1}+x_{2}=2.5,
\end{array}\right.
$$


whose solution is $x_{1}^{*}=1$ and $x_{2}^{*}=1.5$ and we corrupt the involved matrices $\mathbf{A}$ and $\mathbf{b}$ by some small perturbations such that:

$$
[\mathbf{A}]=\left[\begin{array}{ll}
{[1.9999} & 2.0004
\end{array}\right]\left[\begin{array}{l}
3.9989 \\
{[0.0006}
\end{array}\right],[\mathbf{b}]=\left[\begin{array}{c}
{[7.98198 .0228]} \\
{[2.49642 .5039}
\end{array}\right],
$$

We search for the solution $[\mathbf{x}]$ of $[\mathbf{A}][\mathbf{x}]=[\mathbf{b}]$ in the initial domain $\left[\mathbf{x}_{\text {initial }}\right]=$ $\left[\begin{array}{l}{[0,2]} \\ {[0,3]}\end{array}\right]$.

Notice that the determinant of the matrices $\mathbf{A} \in[\mathbf{A}]$ are in $[-2.0013,-1.9977]$ and this interval does not contain 0.

The solutions are:

- Step 1: The FBP algorithm gives $\left[\mathbf{x}_{F B}\right]=\left[\begin{array}{c}{[0.9798,1.0179]} \\ {[1.4862,1.5163]}\end{array}\right]$,

- Step 2: $\left[\mathbf{x}_{\text {new }}\right]=\left[\begin{array}{c}{[0.9869,1.0108]} \\ {[1.4909,1.5116]}\end{array}\right]$

The widths of the solution boxes provide a good indication of the overestimation. We have $w\left(\left[\mathbf{x}_{F B}\right]\right)=(0.0381,0.0301)^{T}$ and $w\left(\left[\mathbf{x}_{\text {new }}\right]\right)=(0.0239,0.0207)^{T}$, and can appreciate a reduction of at least $(0.0301-0.0207) / 0.0301 \times 100=31.2 \%$ (operated along the second component) operated by Step 2 of the algorithm.

In Figure 7 the set of solution boxes of $\{[\mathbf{x}]\}^{1},\{[\mathbf{x}]\}^{2}$ and $\{[\mathbf{x}]\}^{3}$ can be com270 pared. If one drew the convex union of $\{[\mathbf{x}]\}^{1}$, it would result in a larger box than the intersection of the convex union of $\{[\mathbf{x}]\}^{2}$ and the convex union of $\{[\mathbf{x}]\}^{3}$, which is the expected result. 

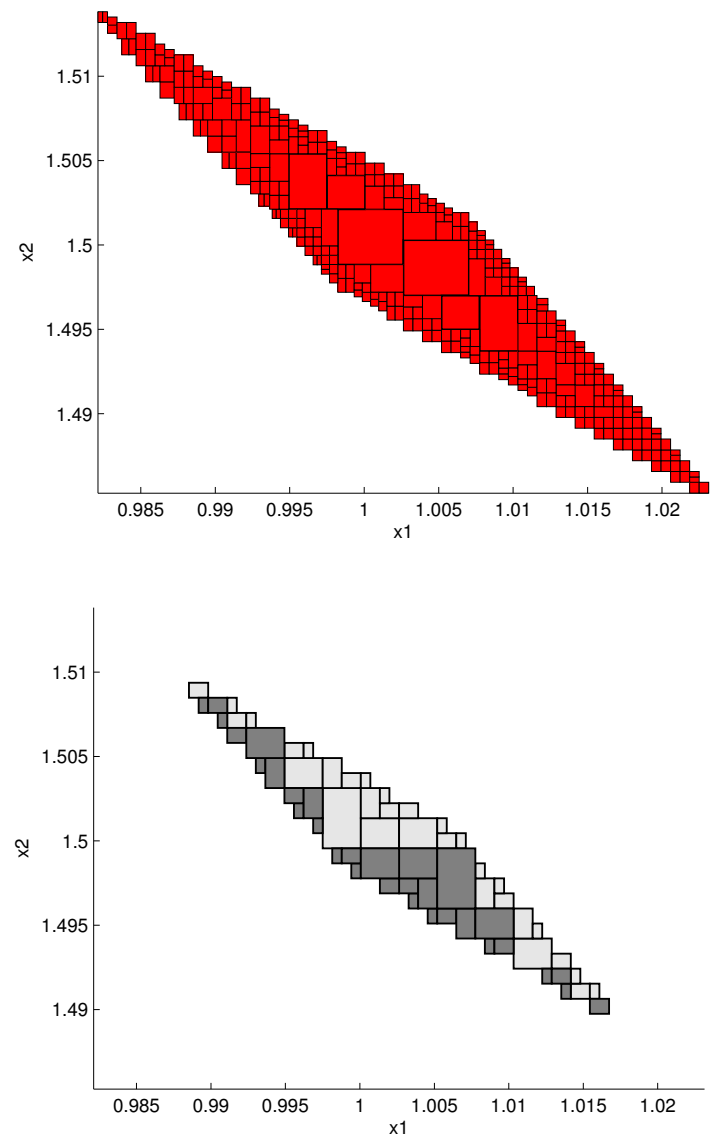

Figure 7: $\{[\mathbf{x}]\}^{1}$ (top), $\{[\mathbf{x}]\}^{2}$ (bottom, light gray) and $\{[\mathbf{x}]\}^{3}$ (bottom, dark gray).

Example 3.2. Let us now consider the following matrices for the linear system of interval equations:

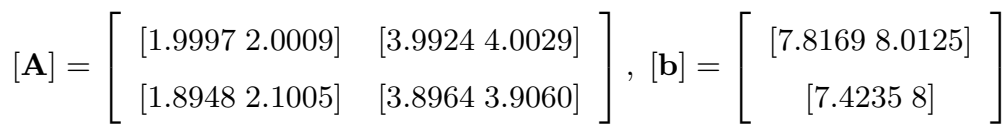

275 Like for the previous example, we search for the solution in $\left[\mathbf{x}_{\text {initial }}\right]=\left[\begin{array}{c}{[0,2]} \\ {[0,3]}\end{array}\right]$.

Notice that the determinant of the matrices $\mathbf{A} \in[\mathbf{A}]$ are in $[-0.6165,0.2832]$, which contains 0 indicating that some of the matrices are singular. 
- Step 1: The FBP algorithm gives $\left[\mathbf{x}_{F B}\right]=\left[\begin{array}{c}{\left[10^{-5}, 2\right]} \\ {[0.9531,2.0069]}\end{array}\right]$,

- Step 2: $\left[\mathbf{x}_{\text {new }}\right]=\left[\begin{array}{c}{\left[10^{-5}, 1.25\right]} \\ {[1.375,1.9905]}\end{array}\right]$.

The widths of the solution boxes are:

$$
\begin{gathered}
w\left(\mathbf{x}_{F B}\right)=(2,1.0538)^{T}, \\
w\left[\mathbf{x}_{\text {new }}\right]=(1.25,0.6155)^{T} .
\end{gathered}
$$

In Figure 8 the set of solution boxes of $\{[\mathbf{x}]\}^{1},\{[\mathbf{x}]\}^{2}$ and $\{[\mathbf{x}]\}^{3}$ can be compared. Like for the previous example, although the convex unions are not reported on the ${ }_{285}$ figures, it is easy to see that the convex union of $\{[\mathbf{x}]\}^{1}$ results in a larger box than the intersection of the convex union of $\{[\mathbf{x}]\}^{2}$ and the convex union of $\{[\mathbf{x}]\}^{3}$.

Remark- When there is a problem of ill-conditioning, classical methods can fail contrary to FBP which shows to be more robust. Indeed, for this example, the Gauss elimination approach fails. 

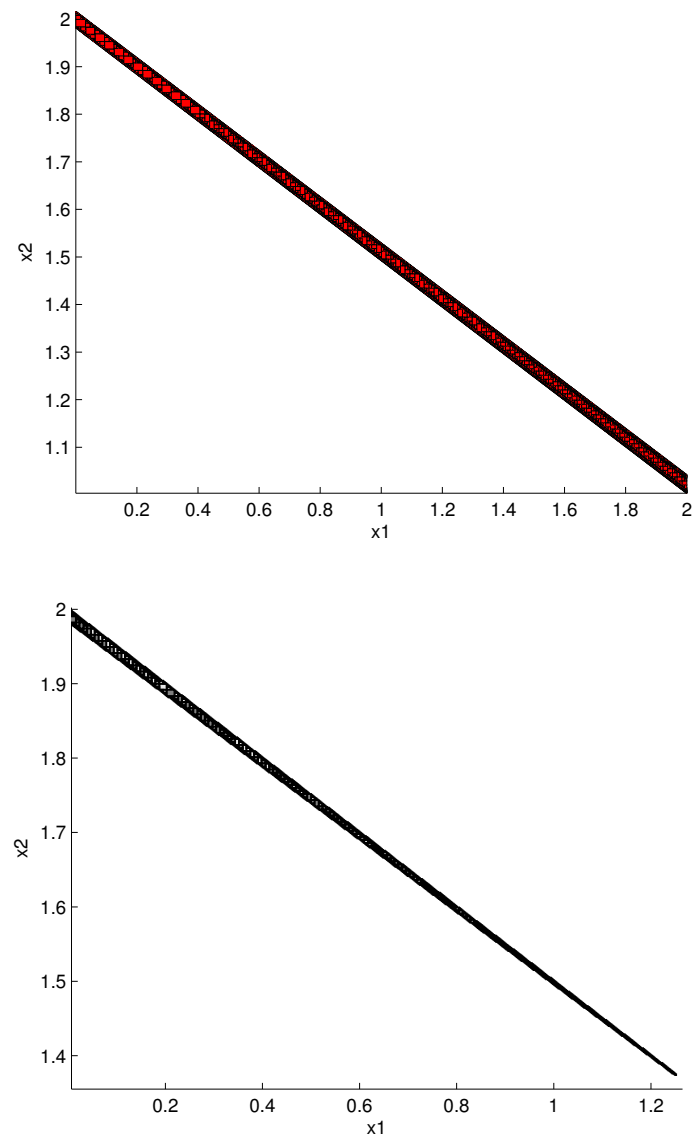

Figure 8: $\{[\mathbf{x}]\}^{1}$ (top), $\{[\mathbf{x}]\}^{2}$ (bottom, light gray) and $\{[\mathbf{x}]\}^{3}$ (bottom, dark gray).

\section{Application to fault detection and isolation}

In this section, we propose an algorithm for fault detection and isolation (FDI) of nonlinear dynamical systems that makes use of the two algorithms presented in sections 2 and 3 . Fault detection and identification are achieved via parameter estimation [25]. The method, based on differential algebra, makes use of relations also known as Analytical Redundancy Relations (ARR) linking the outputs (measurements), the inputs, their derivatives, and the unknown parameters of the system. The ARRs are used to estimate the parameters of the model in a set-membership framework through an analytical solution with the method presented in section 3 The estimation comes 
back to the set inversion problem that solves $[\mathbf{A}][\mathbf{x}]=[\mathbf{b}]$ for $[\mathbf{x}]$, where $[\mathbf{x}]$ represents the parameter blocks and $[\mathbf{A}]$ and $[\mathbf{b}]$ are an interval matrix and interval vector, respectively. The components of matrix $[\mathbf{A}]$ and vector $[\mathbf{b}]$ are in the form of differential polynomials with respect to the system's output, input and their derivatives. These latter signals are estimated as bounded signals thanks to the method provided in section 2. which allows us to evaluate $[\mathbf{A}]$ and vector $[\mathbf{b}]$.

The FDI method is an adaptation to the set-membership framework of the standard fault detection and isolation method based on parameter estimation. It assumes that the nominal parameter values are known within specified error bounds. The system is considered healthy when the parameter estimated values intersect the pre-specified nominal interval box. An anomaly is detected when at least one parameter estimated value has empty intersection with its nominal interval value.

In practice, the nominal parameter interval box is provided as the initial box $\left[\mathbf{x}_{\text {initial }}\right]$ of the set inversion problem that solves $[\mathbf{A}][\mathbf{x}]=[\mathbf{b}]$ for $[\mathbf{x}]$ with the method explained in section 3 . If a solution interval box $\left[\mathbf{x}_{n e w}\right]$ is returned, it means that the parameter estimated values intersect the pre-specified nominal interval box and the system is healthy. On the contrary, if the returned solution is the empty set, the system is faulty. The faulty parameter values can then be determined by replacing $\left[\mathbf{x}_{\text {initial }}\right]$ by a box that is likely to include the faulty parameter values.

\subsection{FDI algorithm}

Let us consider a nonlinear model of the form:

$$
\left\{\begin{array}{l}
\dot{x}(t, p)=f(x(t, p), u(t), p), \\
y(t, p)=h(x(t, p), p), \\
x\left(t_{0}, p\right)=x_{0} \in X_{0}, \\
p \in P \subset \mathcal{U}_{\mathcal{P}}, t_{0} \leq t \leq T,
\end{array}\right.
$$

where :

- $x(t, p) \in \mathbb{R}^{n}$ and $y(t, p) \in \mathbb{R}^{m}$ denote the state variables and the outputs at time $t$ respectively, 
- $u(t) \in \mathbb{R}^{r}$ is the input vector at time $t$; in the case of uncontrolled models, $u(t)$ is equal to 0 ,

- the initial conditions $x_{0}$, if any, are assumed to belong to a bounded set $X_{0}$ and one assumes that $X_{0}$ does not contain equilibrium points of the system,

- the parameter vector $p$ belongs to a connected set $P$ assumed to be included in $\mathcal{U}_{\mathcal{P}}$, where $\mathcal{U}_{\mathcal{P}} \subseteq \mathbb{R}^{p}$ is an a priori known set of admissible parameters; the components of $p$ are denoted $p_{i}$.

- the functions $f$ and $h$ are real and analytic ${ }^{2}$ on $M$, where $M$ is an open set of $\mathbb{R}^{n}$ such that $x(t, p) \in M$ for every $t \in\left[t_{0}, T\right]$ and $p \in P, T$ is a finite or infinite time bound.

ARRs are relations deduced from the model that link the system inputs and outputs and their derivatives. Provided that derivatives can be estimated, ARRs can be evaluated with the measurements and used to estimate the parameters of the model as shown in [25]. For nonlinear model like [10], ARRs can be obtained from the RosenfeldGroebner algorithm implemented in Maple [32].

For any vector $\vartheta$, let us define $\bar{\vartheta}$ to stand for $\vartheta$ and its time derivatives up to some (unspecified) order. Then ARRs can be put in the following form:

$$
w_{i}(\bar{y}, \bar{u}, p)=m_{0, i}(\bar{y}, \bar{u}, p)-\sum_{k=1}^{n_{i}} \gamma_{k}^{i}(p) m_{k, i}(\bar{y}, \bar{u})
$$

where:

- $\left(\gamma_{k}^{i}(p)\right)_{1 \leq k \leq n_{i}}$ are rational in $p$ and so-called "parameter blocks",

- $\gamma_{v}^{i} \neq \gamma_{w}^{i}(v \neq w)$ and $\left(m_{k, i}(\bar{y}, \bar{u})\right)_{1 \leq k \leq n_{i}}$ are differential polynomials with respect to $\bar{y}$ and $\bar{u}$.

The first part of the polynomial $m_{0, i}(\bar{y}, \bar{u}, p)$ is not identically equal to zero and it does not involve the parameter vector $p$.

By using the Rosenfeld-Groebner algorithm with a specific elimination order, it has been proven that the ARRs are linear in some combinations of parameters [33]. The ARRs can be rewritten under the form $0 \in[\mathbf{A}][\mathbf{x}]-[\mathbf{b}]$, where $[\mathbf{x}]$ represents a

\footnotetext{
${ }^{2}$ In particular, $f$ and $h$ are considered infinitely differentiable. This assumption is important for the use of differential algebra.
} 
vector of parameter blocks that we want to estimate. Besides, let us assume that in the initial state the system to diagnose is in a free-fault situation. Data between $n_{c} T_{s}$ and $\left(n_{c}+n_{d}\right) T_{s}$ seconds are used to construct $[\mathbf{A}]$ and $[\mathbf{b}]$, where $n_{d} T_{s}$ denotes the length of the time window during which the parameter estimation takes place. $[\mathbf{A}]$ and

Remark 1. As already mentioned in Section 2.1, the convergence time $t_{c}$ depends upon differentiator parameters, which must be chosen experimentally. Therefore, some tests including healthy and faulty scenarios must be performed to determine $t_{c}$ and the faulty initial box $\left[\mathbf{x}_{f}\right]$. 


\section{Algorithm I}

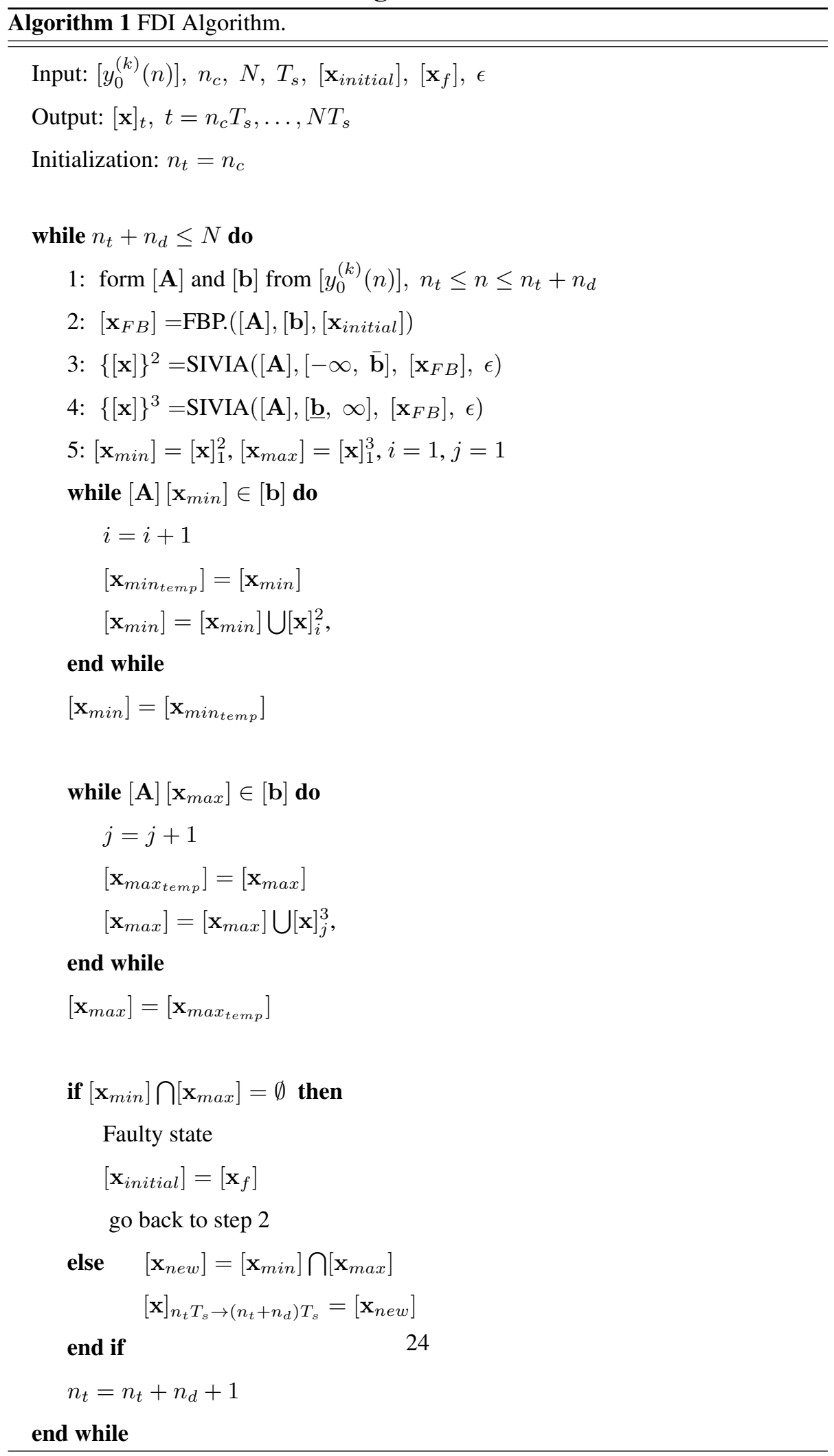




\subsection{Application to a cellular model}

The following example concerns the detection of abnormal situations and identification of some parameters in the process of absorption of glucose oxydase by a cell. Glucose oxydase can be easily detected and measured through spectrophotometric, potentiometric [34] or immunologic techniques [35].

Thus glucose oxydase is easily measurable in practice and is provided continuously to the cell. For this reason, it is assumed to be observed outside the cell.

The model is:

$$
\left\{\begin{array}{l}
\dot{\chi}_{1}=\alpha_{1}\left(\chi_{2}-\chi_{1}\right)-\frac{V_{m} \chi_{1}}{1+\chi_{1}}, \\
\dot{\chi}_{2}=\alpha_{2}\left(\chi_{1}-\chi_{2}\right) \\
\chi_{1}(0)=0.62, \chi_{2}(0)=0 \\
y_{0}=\chi_{1},
\end{array}\right.
$$

where $\chi_{1}\left(\right.$ resp. $\left.\chi_{2}\right)$ is the concentration of glucose oxydase outside (resp. inside) the cell and $\mathbf{p}=\left(\alpha_{1}, V_{m}, \alpha_{2}\right)^{T}$ is the vector of unknown parameters that have to be identified. The normal values of parameters are $\alpha_{1}=0.011, \alpha_{2}=0.02$ and $V_{m}=0.1$. The study has been conducted in simulation in Matlab. The simulated output $y_{0}$ is disturbed by an additive Gaussian noise $e$, which is bounded by $10^{-4}$. The measurements are done at discrete times $n T_{s}, n=0, \ldots, N$, on the interval $[0,60]$ with a sampling period equal to $1 \mathrm{~s} . N$ is the total number of sampling times $3^{3}$

The following ARR $R\left(y_{0}\right)$ is obtained with the Rosenfeld-Gröbner algorithm implemented in Maple 16 [32]:

$$
R\left(y_{0}\right)=\ddot{y}_{0}\left(1+y_{0}\right)^{2}+\gamma_{1} \dot{y}_{0}\left(1+y_{0}\right)^{2}+\gamma_{2} y_{0}\left(1+y_{0}\right)+\gamma_{3} \dot{y}_{0},
$$

where $\gamma_{1}=\alpha_{1}+\alpha_{2}, \gamma_{2}=\alpha_{2} V_{m}$ and $\gamma_{3}=V_{m}$.

By using the Rosenfeld-Groebner algorithm with a specific elimination order, the obtained ARR $R\left(y_{0}\right)$ is linear in some combinations of parameters (parameter blocks)

\footnotetext{
${ }^{3}$ Using this model and assumptions for emulation of a biological system may rise some issues, in particular the assumption of bounded and known measurement noise characteristics should be carefully assessed. Recent biological experiments suggest that this can be quite reasonable in some cases.
} 

and $\mathbf{b}=-\ddot{y}_{0}\left(1+y_{0}\right)^{2}$ and the unknown vector of blocks of parameters is $\mathbf{x}=$ $\left[\gamma_{1}, \gamma_{2}, \gamma_{3}\right]^{T}=\left[\alpha_{1}+\alpha_{2}, \alpha_{2} V_{m}, V_{m}\right]^{T}$

As it can be seen in Equation (13), the first and second derivatives of $y_{0}$ are in400 volved. After simulation tests, the parameters of the HOSM differentiator are chosen such that $\lambda_{0}=3, \lambda_{1}=0.2, \lambda_{2}=0.1, \eta_{1}=0.0427$ and $\eta_{2}=0.0038$.

The involved intervals $\left[y_{0}^{k}(n)\right]$ with $k=0,1,2, \ldots$ and $n=0, \ldots, 60$ are computed by filtering the intervals found by the HOSM differentiator. A second-order low-pass Butterworth zero-phase filter of cutoff frequency $5 \mathrm{~Hz}$ is used. The convergence time is considered as $t_{c}=5 \mathrm{~s}$. Algorithm I is hence used to detect and identify abnormalities occurring between 5 and 60 seconds.

Two abnormal scenarios are considered:

Scenario 1: $V_{m}$ jumps from its normal value 0.1 to 0.15 at $t=22 \mathrm{~s}$.

Scenario 2: $\alpha_{2}$ jumps from its normal value 0.02 to 1.5 at $t=17 \mathrm{~s}$.

In the FDI algorithm, we choose the bisection precision threshold as $\epsilon=0.05$, $n_{d}=2$, and

$$
\left[\mathbf{x}_{\text {initial }}\right]=\left[\begin{array}{c}
{\left[10^{-5}, 0.04\right]} \\
{\left[10^{-5}, 0.003\right]} \\
{\left[10^{-5}, 0.2\right]}
\end{array}\right]
$$

Case of scenario 1: the abnormal value is detected at $t=1.01 \mathrm{~s}$ after its occurrence. Once the abnormality value is detected, the estimation algorithm is initialized with:

$$
\left[\mathbf{x}_{f}\right]=\left[\begin{array}{c}
{[0.031,0.04]} \\
{\left[10^{-5}, 0.006\right]} \\
{\left[10^{-5}, 0.4\right]}
\end{array}\right]
$$


and we obtain at $t=26 \mathrm{~s}$, the box:

$$
\left[\mathbf{x}_{\text {new }}\right]=\left[\begin{array}{c}
{[0.031,0.04]} \\
{\left[10^{-5}, 0.006\right]} \\
{[0.1271,0.1985]}
\end{array}\right]
$$

which leads to the following parameter set values $\alpha_{1}=[0,0.0399], \alpha_{2}=[0,0.0473]$ and $V_{m}=[0.1271,0.1985]$.

The interval values for $\alpha_{1}$ and $\alpha_{2}$ contain the normal values whereas the one for $V_{m}$ contains the abnormal value, hence the abnormality is confirmed. These results are presented in Figure 9. In the graphs providing the estimations for $\alpha_{1}, \alpha_{1}$, and $V_{m}$, the gap in the data comes from switching from the detection to the identification algorithm.
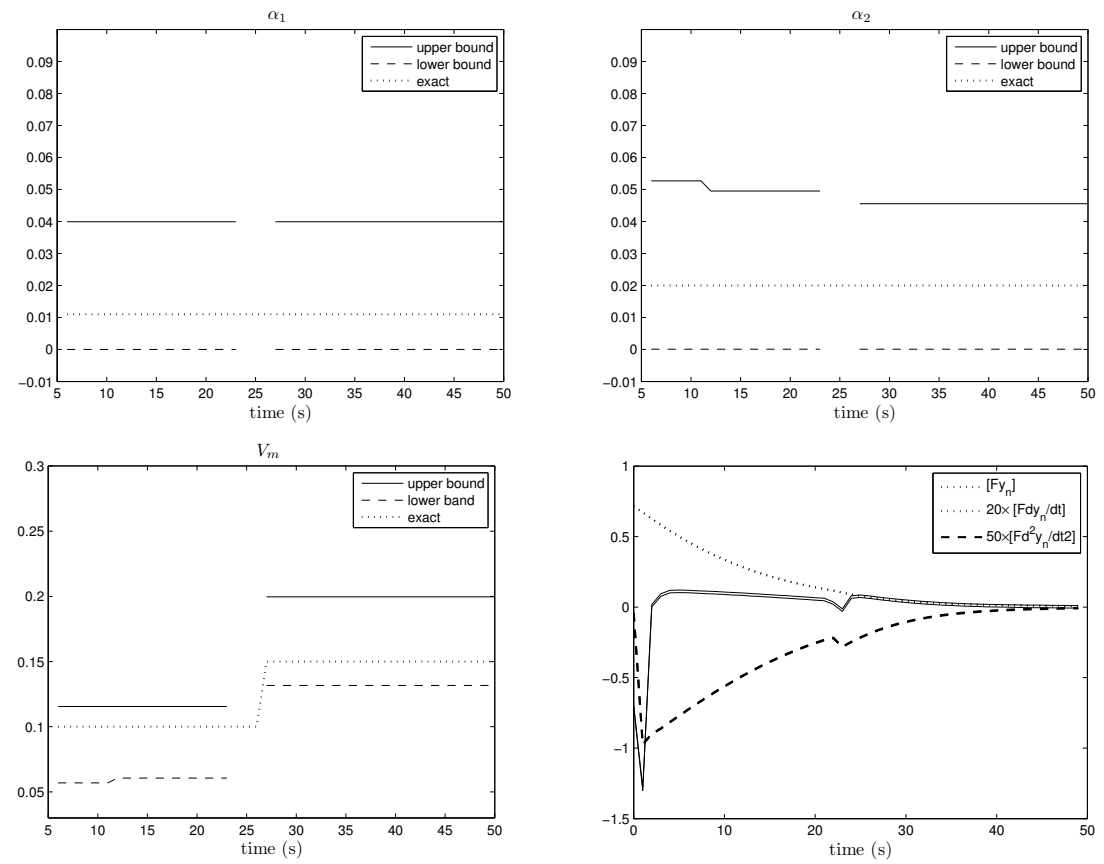

Figure 9: Abnormality detection and identification for scenario 1 . The bottom right graph provides the signal $y_{0}$ and the estimation of its $1^{\text {st }}$ and $2^{\text {nd }}$ derivatives used in the FDI algorithm.

Case of scenario 2: the abnormal value is detected in $t=2.59 \mathrm{~s}$ after its occurence. 
Once the abnormality is detected, the estimation algorithm is initialized with:

$$
\left[\mathbf{x}_{f}\right]=\left[\begin{array}{c}
{\left[10^{-5}, 2\right]} \\
{\left[10^{-1}, 0.18\right]} \\
{\left[10^{-1}, 0.114\right]}
\end{array}\right],
$$

and gives at $t=23 \mathrm{~s}$ the following estimation:

$$
\left[\mathbf{x}_{\text {new }}\right]=\left[\begin{array}{c}
{[1.0192,1.781]} \\
{[0.1,0.18]} \\
{[0.0726,0.114]}
\end{array}\right]
$$

thus $\alpha_{1}=[0,0.9039], \alpha_{2}=[0.8771,2.4794]$ and $V_{m}=[0.0726,0.14]$. At $t=52 \mathrm{~s}$, the algorithm gives the following estimation:

$$
\left[\mathbf{x}_{\text {new }}\right]=\left[\begin{array}{c}
{[1.0177,1.4445]} \\
{[0.1,0.1378]} \\
{[0.0752,0.114]}
\end{array}\right]
$$

420

and we obtain the parameter set values $\alpha_{1}=[0,0.5674], \alpha_{2}=[0.8771,1.8325]$ and $V_{m}=[0.0752,0.114]$. The interval values for $\alpha_{1}$ and $V_{m}$ contain the normal values whereas the one for $\alpha_{2}$ contains the abnormal value, hence the abnormality is confirmed. These results are presented in Figure 10 

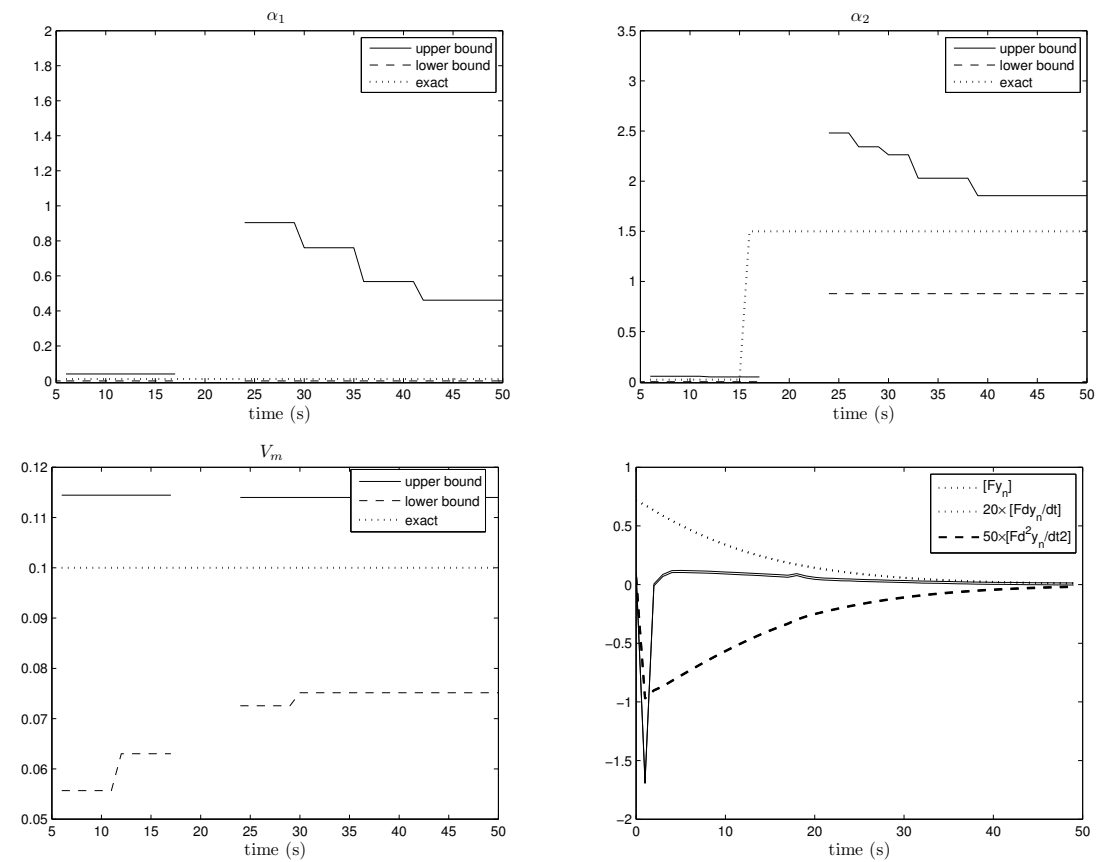

Figure 10: Abnormality detection and identification for scenario 2. The bottom right graph provides the signal $y_{0}$ and the estimation of its $1^{s t}$ and $2^{\text {nd }}$ derivatives used in the FDI algorithm.

Remark - Notice that if the initial box for the abnormal situation does not contain the exact value of the abnormal parameter, the estimation solution is empty. Thus the conclusion is that the initial box must be changed.

\section{Conclusion}

This paper considers two problems which take often part of the solutions proposed for SM estimation and its applications to engineering problems. The first one is the problem of estimating high order derivatives of noisy signals. Considering a low pass signal corrupted by bounded noise, the problem of the HOSM differentiator that provides wide enclosures is improved by smoothing the upper and lower boundaries. It is shown that this enhancement enables one to provide useful enclosures of relatively high-order derivatives. The second problem is encountered when solving ill-conditioned linear interval equations. Initializing the SIVIA based optimization algorithm by Forward Backward Propagation iterations is shown to reduce the overesti- 
mation of the linear interval system solution box. This property is specially appreciated in the case of linear systems with badly conditioned matrices. It is also shown that formulating the problem in two equivalent subproblems and combining their solutions interestingly reduces the overestimation of the solution.

The two proposed methods are integrated in a recursive SM estimation algorithm for FDI of nonlinear systems with bounded uncertainty. The application to a cell exchange model illustrates the efficiency of the algorithm.

It should be noticed that the two problems that find improved solutions in this paper are quite generic and take part in the methods providing solutions to many engineering tasks. These two problems are quite generic and then our solutions can be used in many other similar engineering context

In particular, estimating derivatives may be an elegant solution to turn a differential problem into an algebraic form, hence avoiding the difficulty of integration in a setmembership framework.

\section{Acknowledgement}

This work was supported by the French National Research Agency (ANR) in the framework of the project ANR-11-INSE-006 (MAGIC-SPS).

\section{References}

[1] E. Gelso, G. Biswas, S. Castillo, J. Armengol, A comparison of two methods for fault detection: a statistical decision, and an interval-based approach, in: Proceedings of 19th International Workshop on Principles of Diagnosis DX, Blue Montains, Australia, 2008, pp. 261-268.

[2] L. Jaulin, I. Braems, M. Kieffer, E. Walter, Nonlinear state estimation using forward-backward propagation of intervals in an algorithm, Scientific Computing, Validated Numerics, Interval Methods (2001) 191-204.

[3] M. Milanese, C. Novara, Nonlinear Set Membership prediction of river flow, Systems \& Control Letters 53 (1) (2004) 31-39. 
[4] M. Kieffer, L. Jaulin, E. Walter, D. Meizel, Guaranteed mobile robot tracking using interval analysis, in: Proceedings of MISC'99, Workshop on Application of Interval Analysis to System and Control, Girona, Spain, 1999, pp. 347-360.

[5] R. Moore, Interval analysis, Prentice-Hall, Englewood Cliffs, 1966.

[6] L. Jaulin, M. Kieffer, O. Didrit, E. Walter, Applied Interval Analysis, with examples in parameter and state estimation, Robust control and robotics, Springer, Londres, 2001.

[7] M. Maïga, C. Combastel, N. Ramdani, L. Travé-Massuyès, Nonlinear hybrid reachability using set integration and zonotope enclosures, in: Proceedings of the 13th European Control Conference, Strasbourg, France, 2014, pp. 234-239.

[8] S. Lesecq, A. Barraud, K. Dinh, Numerical accurate computations for ellipsoidal state bounding, in: Proceedings of the 11th IEEE Mediterranean Conference on Control and Automation, Rhodes, Greece, 2003.

[9] A. Ingimundarson, J. Manuel Bravo, V. Puig, T. Alamo, P. Guerra, Robust fault detection using zonotope-based set-membership consistency test, International Journal of Adaptive Control And Signal Processing 23 (4) (2009) 311-330.

[10] C. De Boor, A practical Guide to Splines, Springer, New York, 1978.

[11] S. Ibrir, S. Diop, A numerical procedure for filtering and efficient high-order signal differentiation, International Journal of Applied Mathematics and Computer Science 14, No. 2 (2004) 201-208.

[12] M. Fliess, M. Mboup, H. Mounier, H. Sira-Ramirez, Questioning some paradigms of signal processing via concret examples, in: Proceedings of Summer School: Fast Estimation Method in Automatic Control and Signal Processing, Paris, 2005.

[13] M. Mboup, C. Join, M. Fliess, Numerical differentiation with annihilator in noisy environment, Numerical Algorithms 50, No. 4 (2009) 439-467. 
[14] N. Verdière, L. Denis-Vidal, G. Joly-Blanchard, A new method for estimating derivatives based on a distribution approach, Numerical Algorithms 61, Issue 1 (September 2012) 163-186.

[15] A. Levant, Robust exact differentiation via sliding mode technique, Automatica 34 (1998) 379-384.

[16] A. Levant, Higher-order sliding modes, differentiation and output-feedback control, International Journal of Control 76 (2003) 924-941.

[17] C. Jauberthie, N. Verdière, L. Travé-Massuyès, Set-membership identifiability: definitions and analysis, in: Proceedings of 18th IFAC World Congress, Milan, Italie, 2011, pp. 12024-12029.

[18] R. Seydou, R. Raïssi, A. Zolghadri, D. Efimov, Actuator fault diagnosis for flat systems: A constraint satisfaction approach, International Journal of Applied Mathematics and Computer Science 23, No. 1 (2013) 171-181.

[19] J. Rohn, V. Kreinovich, Computing exact component wise bounds on solutions of linear systems with interval data is np-hard, SIAM Journal on Matrix Analysis and Applications 16 (1995) 415-420.

[20] H. Nguyen, N. Revol, Solving and certifying the solution of a linear system, Reliable computing (2) (2011) 120-131.

[21] E. Hansen, Bounding the solution of interval linear equations, SIAM Journal on Numerical Analysis 29 (1992) 1493-1503.

[22] J. Rohn, Cheap and tight bounds: the recent result by E. Hansen can be made more efficient, Interval computations (4) (1993) 13-21.

[23] G. Chabert, L. Jaulin, Contractor programming, Artifical Intelligence 173 (2009) $1079-1100$.

[24] L. Jaulin, E. Walter, Set inversion via interval analysis for nonlinear boundederror estimation, Automatica 29 (1993) 1053-1064. 
[25] C. Jauberthie, N. Verdière, L. Travé-Massuyès, Fault detection and identification relying on set-membership identifiability, Annual Reviews in Control 37, No. 1 (2013) 129-136.

[26] A. d'Onofrio, Bounded Noises in Physics, Biology, and Engineering, Springer, 2013.

[27] F. Carbajal, A. Contreras, A. Valderrábano González, G. Silva Navarro, Asymptotic differentiation of signals in the trajectory tracking control of a differentially flat nonlinear magnetic suspension system, Revista Facultad de Ingeniería (66) (2013) 70-81.

[28] H. Hammouri, B. Targui, F. Armanet, High gain observer based on a triangular structure, International Journal of Robust and Nonlinear Control 12(6) (2002) 497-518.

[29] A. Levant, Higher order sliding modes and arbitrary order exact robust differentiation, in: Proceedings of European Control Conference, Porto, Portugal, 2001, pp. 996-1001.

[30] A. Oppenheim, R. Schafer, Discrete-time signal processing, Prentice Hall Signal Processing Series, third edition, 2010.

[31] J. Rohn, Systems of linear interval equations, Linear Algebra and its Applications 126 (1989) $39-78$.

[32] F. Boulier, D. Lazard, F. Ollivier, M. Petitot, Computing representations for the radicals of a finitely generated differential ideals, Technical report IT306, LIFL, University of Lille.

[33] L. Denis-Vidal, G. Joly-Blanchard, C. Noiret, M. Petitot, An algorithm to test identifiability of non-linear systems, in: Proceedings of 5th IFAC Non-Linear Control Systems, Vol. 7, St Petersburg, Russia, 2001, pp. 174-178.

[34] A. Aubrée-Lecat, C. Hervagault, A. Delacour, P. Beaude, C. Bourdillon, M.H. Remy, Direct electrochemical determination of glucose oxidase in biological samples, Analytical Biochemistry 178 (2) (1989) 427 - 430. 
[35] C. Bellefontaine, F. Josse, M. Domurado, D. Domurado, Immunoassay for native enzyme quantification in biological samples, Applied Biochemistry and Biotechnology 48, No. 2 (1994) 117-123. 